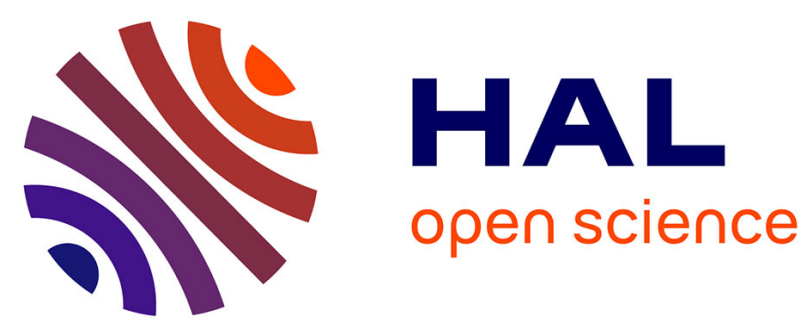

\title{
Set Adaptive Observers for Linear Parameter-Varying Systems: Application to Fault Detection
}

\author{
Denis Efimov, Tarek Raissi, Ali Zolghadri
}

\section{To cite this version:}

Denis Efimov, Tarek Raissi, Ali Zolghadri. Set Adaptive Observers for Linear Parameter-Varying Systems: Application to Fault Detection. Journal of Dynamic Systems, Measurement, and Control, 2014, 136 (2), pp.7. 10.1115/1.4025797 . hal-00843639

\section{HAL Id: hal-00843639 \\ https://hal.inria.fr/hal-00843639}

Submitted on 11 Jul 2013

HAL is a multi-disciplinary open access archive for the deposit and dissemination of scientific research documents, whether they are published or not. The documents may come from teaching and research institutions in France or abroad, or from public or private research centers.
L'archive ouverte pluridisciplinaire HAL, est destinée au dépôt et à la diffusion de documents scientifiques de niveau recherche, publiés ou non, émanant des établissements d'enseignement et de recherche français ou étrangers, des laboratoires publics ou privés. 


\title{
SET ADAPTIVE OBSERVERS FOR LPV SYSTEMS: APPLICATION TO FAULT DETECTION
}

\author{
Denis Efimov ${ }^{1}$, Tarek Raïssi ${ }^{2}$, Ali Zolghadri ${ }^{3}$ \\ ${ }^{1}$ Non-A project at INRIA - LNE, Parc Scientifique de la Haute Borne, \\ 40 avenue Halley, Bât.A Park Plaza, 59650 Villeneuve d'Ascq, France \\ Denis.Efimov@inria.fr \\ ${ }^{2}$ Conservatoire National des Arts et Métiers, Département EASY, Cedric - laetitia \\ 292, Rue St-Martin, case 2D2P10, 75141 Paris Cedex 03 \\ Tarek.Ra1ssi@cnam.fr \\ ${ }^{3}$ University of Bordeaux, IMS-lab, Automatic control group \\ 351 cours de la libération, 33405 Talence, France \\ Ali.Zolghadri@ims-bordeaux.fr
}

\begin{abstract}
This paper deals with the problem of joint state and parameter estimation based on a set adaptive observer design. The problem is formulated and solved for an LPV (Linear ParameterVarying) system. The resolution methodology avoids the exponential complexity obstruction usually encountered in the set-membership parameter estimation. A simulation example is presented to illustrate the efficiency of the proposed approach.
\end{abstract}

Keywords: Adaptive observers; LPV systems; fault detection.

\section{Introduction}

Let a nonlinear system be described by:

$$
\dot{\mathbf{x}}=\mathbf{f}(t, \mathbf{x}, \mathbf{u}, \mathbf{d}), \mathbf{y}=\mathbf{h}(\mathbf{x})+\mathbf{v},
$$

where $\mathbf{x} \in R^{n}, \mathbf{u} \in R^{m}, \mathbf{d} \in R^{l}, \mathbf{y} \in R^{p}, \mathbf{v} \in R^{p}$ are respectively the state, the input, the disturbance, the output and the measurement noise, $t \in R_{+}$and the functions $\mathbf{f}, \mathbf{h}$ are continuous with respect to all arguments and differentiable with respect to $\mathbf{x}$ and $\mathbf{u}$.

Observer design for nonlinear systems has been an area of intensive research during the last two decades. See for instance [3], [25] and the references therein. Typically, the observer design problem is solvable if the system model can be transformed into a canonical form [3], which may be a hard assumption to satisfy in many applications. Another appealing approach is based on the LPV transformation of (1) [7], [17], [21], [31]:

$$
\dot{\mathbf{x}}=\mathbf{A}(\rho(t)) \mathbf{x}+\mathbf{B}(\rho(t)) \mathbf{u}, \mathbf{y}=\mathbf{C}(\rho(t)) \mathbf{x}+\mathbf{v},
$$

where the scheduling parameter vector $\rho \in \mathcal{P}$ is considered to be time-varying (measured or estimated during system operation) or unknown with known bounds, $\mathcal{P}$ is a set of functions that re- 
main in a compact real subspace. The system (2) is an equivalent representation of (1), in the sense that all trajectories of (1) remain within the set of trajectories generated by (2) [17], [22], [31], [32], [35]. The basic idea is to replace the nonlinear complexity of the model (1) by enlarged parametric variations in the LPV model (2), which simplifies the design of an observer for (1).

On the other hand, interval observers have received much attention recently to solve a number of state and parameter estimation problems. One can distinguish mainly three approaches to interval state estimation in a bounded error context: the prediction/correction mechanism [15], [27]; the approach based on comparison theorem [20], [24]; and the closed loop interval observers with cooperative estimation error dynamics [2], [14], [23]. The latter has been extended in [28] for nonlinear systems having LPV representations with known minorant and majorant matrices for (2). However, these interval state estimators are only efficient when the parameter uncertainties are not large.

In the open literature, the general problem of joint state and parameter estimation has not been fully studied for (1) in a bounded error context. Attempts to take into account the uncertain parameters in set-membership framework were made in [16], [18], [27] applying some computationally time-consuming numerical techniques with an exponential complexity (including the branch-andbound method) with respect to the parameter vector dimension.

The joint state and parameter estimation is addressed in the adaptive observer framework [10], [11], [34], [37], [39], [40]. Typically the adaptive observer design methods can be applied for the systems in the output canonical form, i.e. when the system matrices and functions dependent on measured signals. This is not the case for LPV systems with not measured vector of scheduling parameters.

In the following, the methodology proposed in [28] is extended using the theory of adaptive observers to deal with joint state and parameter estimation which can be applied to high dimensional systems with large parametric uncertainties. The main distinguishing feature with respect to works [2], [14], [23], [28] is that the cooperativity property of the state observers is not inherited by the adaptive counterpart. The advantage of the proposed approach is that no bisection is needed in the 
parameter estimation procedure and the complexity of the algorithm is not exponential, making it suitable for dealing with high dimensional systems. Contrarily the conventional approaches to adaptive observer design [10], [11], [34], [37], [39], [40], the developed method can be applied to the LPV systems with not measured vector of scheduling parameters. Potential application to fault detection is also investigated. It is shown that the computed estimates of unknown parameters improve robustness of fault detection and the false alarm rate. The formal description of the studied problem is given in the next section.

\section{Problem statement}

Assume that the system (1) can be represented in the following form:

$$
\dot{\mathbf{x}}=\mathbf{A}(\rho(t)) \mathbf{x}+\mathbf{B}(\rho(t)) \mathbf{u}+\phi(\mathbf{y})+\mathbf{G}(\mathbf{y}) \boldsymbol{\theta}, \mathbf{y}=\mathbf{C} \mathbf{x}, \mathbf{y}_{v}=\mathbf{y}+\mathbf{v}
$$

where $\mathbf{x} \in X \subset R^{n}, \mathbf{u} \in U \subset R^{m}, \mathbf{y} \in Y \subset R^{p}$ are the state, the input and the output vectors; $\boldsymbol{\theta} \in \Theta \subset R^{q}$ is the vector of uncertain parameters; $\mathbf{v} \in V \subset R^{p}$ is the measurement noise; $\mathbf{y}_{v}$ is the vector of noisy measurements of the system (3), $\rho \in \Upsilon \subset R^{r}$ is some scheduling parameter vector. The compact sets $X, U, Y, V, \Theta$ and $\Upsilon$ are given a priori, and it is assumed that there are some constant vectors $\mathbf{x}_{m}, \mathbf{x}_{M} \in R^{n}$ such that $\mathbf{x}_{m} \leq \mathbf{x} \leq \mathbf{x}_{M}$ for all $\mathbf{x} \in X$ (for the vectors $\mathbf{x}, \boldsymbol{\xi}$ the inequality $\mathbf{x} \leq \xi$ is understood elementwise). The vector function $\phi$ and the columns of the matrix function $\mathbf{G}$ are assumed to be locally Lipschitz continuous, $\mathbf{C}$ is some constant matrix of an appropriate dimension. It is worth noting that linearization of the terms depending on the output $\mathbf{y}$ is not necessary. The majorant matrices $\mathbf{A}_{m}, \mathbf{A}_{M}, \mathbf{B}_{m}, \mathbf{B}_{M}$ are given such that $\mathbf{A}_{m} \prec \mathbf{A}(\rho) \prec \mathbf{A}_{M}$, $\mathbf{B}_{m} \prec \mathbf{B}(\rho) \prec \mathbf{B}_{M}$ for all $\rho \in \Upsilon$ (the inequality $\mathbf{A} \prec \mathbf{B}$ for matrices $\mathbf{A}$, $\mathbf{B}$ with dimension $n \times m$ is understood elementwise $A_{i, j} \leq B_{i, j}, i=\overline{1, n}, j=\overline{1, m}$ ). Note that since $\mathbf{y} \in Y$ and $\mathbf{v} \in V$, there exist constants $k_{\phi}>0, k_{G}>0$ such that $\left|\phi(\mathbf{y})-\phi\left(\mathbf{y}_{v}\right)\right| \leq k_{\phi}|\mathbf{v}|$ and $\left|\mathbf{G}(\mathbf{y})-\mathbf{G}\left(\mathbf{y}_{v}\right)\right| \leq k_{G}|\mathbf{v}|$.

It is required to design an observer for (3) that in the noise-free case provides interval observation of the unmeasured components of the vector $\mathbf{x}$ and estimates the set of admissible values for 
$\boldsymbol{\theta}$. For any $\mathbf{v}(t) \in V, t \geq 0$ the observer solutions have to be bounded.

The reminder of the paper is organized as follows. After introducing the preliminaries in Section 3, a methodology for adaptive observer design is presented in sections 4 and 5 . The application to the fault detection problem is studied in Section 6.

\section{Preliminaries}

\subsection{Cooperativity of dynamical systems}

The system

$$
\dot{\mathbf{x}}=\mathbf{f}(t, \mathbf{x}), \mathbf{x} \in X, t \geq 0
$$

is called cooperative if $\partial f_{i}(t, \mathbf{x}) / \partial x_{j} \geq 0$ for all $1 \leq i \neq j \leq n, t \geq 0$ and $\mathbf{x} \in X$ [33]. Define the solution of (4) as $\mathbf{x}\left(t, \mathbf{x}_{0}\right)$ for the initial condition $\mathbf{x}(0)=\mathbf{x}_{0}$. For the cooperative system (4), if $\mathbf{x}_{0} \leq \xi_{0} \Rightarrow \mathbf{x}\left(t, \mathbf{x}_{0}\right) \leq \mathbf{x}\left(t, \xi_{0}\right)$ for all $t \geq 0$ [33]. A matrix $\mathbf{A}$ with dimension $n \times n$ is called Metzler if $A_{i, j} \geq 0$ for $1 \leq i \neq j \leq n$. A Metzler matrix $\mathbf{A}$ is Hurwitz if and only if there exists a vector $\mathbf{p} \in R^{n}, \mathbf{p}>0$ such that $\mathbf{p}^{T} \mathbf{A}<0$. If $\mathbf{A}$ is Metzler and Hurwitz in the system $\dot{\mathbf{s}}(t)=\mathbf{A} \mathbf{s}(t)+\mathbf{r}(t), \mathbf{s} \in R^{n}, \mathbf{r} \in R^{n}, t \geq 0$, then the properties $\mathbf{s}(0) \geq 0, \mathbf{r}(t) \geq 0$ for all $t \geq 0$ imply $\mathbf{s}(t) \geq 0$ for $t \geq 0$ and, conversely, $\mathbf{s}(0) \leq 0, \mathbf{r}(t) \leq 0$ for all $t \geq 0$ ensure $\mathbf{s}(t) \leq 0$ for $t \geq 0$. The system (4) is called competitive if $\partial f_{i}(t, \mathbf{x}) / \partial x_{j} \leq 0$ for all $1 \leq i \neq j \leq n, t \geq 0$ and $\mathbf{x} \in X$, the competitive systems behave like cooperative in the backward time [33].

\subsection{Persistency of excitation}

The Lebesgue measurable and square integrable matrix function $\mathbf{R}: R \rightarrow R^{l_{1} \times l_{2}}$ with dimension $l_{1} \times l_{2}$ admits a $(\ell, \vartheta)$-persistency of excitation (PE) condition, if there exist constants $\ell>0, \vartheta>0$ such that $\int_{t}^{t+\ell} \mathbf{R}(s) \mathbf{R}(s)^{T} d s \geq \vartheta \mathbf{I}_{l_{1}}$ for any $t \in R_{+}$, where $\mathbf{I}_{l_{1}}$ is the identity matrix of dimension $l_{1} \times l_{1}[1],[38]$. 
L e m m a 1 [10]. Consider the time-varying linear dynamical system

$$
\dot{\mathbf{p}}=-\Gamma \mathbf{R}(t) \mathbf{R}(t)^{T} \mathbf{p}+\mathbf{b}(t), t_{0} \in R_{+},
$$

where $\mathbf{p} \in R^{l_{1}}, \Gamma$ is a positive definite symmetric matrix of dimension $l_{1} \times l_{1}$ and the functions $\mathbf{R}: R_{+} \rightarrow R^{l_{1} \times l_{2}}, \mathbf{b}: R_{+} \rightarrow R^{l_{1}}$ are Lebesgue measurable and essentially bounded, and $\mathbf{R}$ is $(\ell, \vartheta)-$ PE for some $\ell>0, \vartheta>0$. Then, for any initial condition $\mathbf{p}\left(t_{0}\right) \in R^{l_{1}}$, the solution of $(5)$ is defined for all $t \geq t_{0}$ and satisfies ( $\gamma>0$ is the smallest eigenvalue of the matrix $\Gamma$ )

$$
|\mathbf{p}(t)| \leq\left|\mathbf{p}\left(t_{0}\right)\right| e^{-0.5 \gamma \vartheta \ell^{-1}\left(t-t_{0}-\ell\right)}+\left(1+2 \vartheta^{-1} \gamma^{-1} e^{-0.5 \vartheta \gamma}\right) \ell\|\mathbf{b}\|,
$$

where $\|\mathbf{b}\|=$ ess $\sup _{t \geq 0}|\mathbf{b}(t)|$ and $|\mathbf{p}|$ is the usual Euclidean norm.

\subsection{Averaging of a slow dynamics}

Let expression "for $\varepsilon \downarrow 0$ " mean that there exists an $\varepsilon_{o}>0$ such that the relevant statement holds for all $\varepsilon \in\left(0, \varepsilon_{o}\right]$. Recall that for a function $\varphi: I_{\varepsilon} \times R_{+} \rightarrow R, I_{\varepsilon} \subset R_{+}$the symbols $O(\cdot)$ and $o(\cdot)$ can be defined as follows.

1. We say that $\varphi(t, \varepsilon)=O[\delta(\varepsilon)]$ for $\varepsilon \downarrow 0$ if there exist constants $\varepsilon_{o}>0$ and $k>0$ such that $|\varphi(t, \varepsilon)| \leq k|\delta(\varepsilon)|$ for all $t \in I_{\varepsilon}$, for $0<\varepsilon<\varepsilon_{o}$.

2. We say that $\varphi(t, \varepsilon)=o[\delta(\varepsilon)]$ for $\varepsilon \downarrow 0$ if $\lim _{\varepsilon \rightarrow 0}|\varphi(t, \varepsilon)| / \delta(\varepsilon)=0$ uniformly for $t \in I_{\varepsilon}$.

Following [30], consider a vector field $\mathbf{f}(\mathbf{x}, t)$ with $\mathbf{f}: R^{n} \times R_{+} \rightarrow R^{n}$, Lipschitz continuous in $\mathbf{x}$ on $D \subset R^{n}, t \in R_{+}$; continuous for all $(\mathbf{x}, t) \in D \times R_{+}$. For a such $\mathbf{f}$ define the average

$$
\overline{\mathbf{f}}(\mathbf{x})=\lim _{T \rightarrow+\infty} T^{-1} \int_{0}^{T} \mathbf{f}(\mathbf{x}, t) d t .
$$

If $\overline{\mathbf{f}}$ exists and the limit is uniform in $\mathbf{x}$ on compact sets $K \subset D$, then $\mathbf{f}$ is called a KBM-vector field (KBM stands for Krylov, Bogoliubov and Mitropolsky).

Proposition 1 [30]. Consider the initial value problem

$$
\dot{\mathbf{x}}=\varepsilon \mathbf{f}(\mathbf{x}, t), \mathbf{x}(0)=\mathbf{a},
$$


with $\mathbf{a}, \mathbf{x} \in D \subset R^{n}$. Suppose $\mathbf{f}$ is a KBM-vector field producing the averaged equation

$$
\dot{\mathbf{z}}=\varepsilon \overline{\mathbf{f}}(\mathbf{z}), \mathbf{z}(0)=\mathbf{a},
$$

where $\mathbf{z}=0$ is an asymptotically stable critical point in the linear approximation, $\overline{\mathbf{f}}$ is moreover continuously differentiable in $D$ and has a domain of attraction $D^{o} \subset D$. Then for any compact $K \subset D^{o}$ and all $\mathbf{a} \in K$

$$
\mathbf{x}(t)-\mathbf{z}(t)=O[\delta(\varepsilon)], 0 \leq t<+\infty
$$

with $\delta(\varepsilon)=o(1)$ in the general case and $O(\varepsilon)$ provided that $\mathbf{f}$ is periodic in $t$.

\section{Interval parameters estimation}

In this section a set adaptive observer is presented, which estimates the interval of admissible values for the vector of unknown parameters $\boldsymbol{\theta}$. The state $\mathbf{x}$ estimator will be given in Section 5 .

To design a set adaptive observer we need the following assumption.

Assumption 1. There exist two gains $\mathbf{L}_{m}, \mathbf{L}_{M}$ such that the matrices $\mathbf{A}_{m}-\mathbf{L}_{m} \mathbf{C}$ and $\mathbf{A}_{M}-\mathbf{L}_{M} \mathbf{C}$ are Hurwitz and Metzler, $0 \prec \mathbf{C}$ and for all $\mathbf{y} \in Y, \mathbf{v} \in V$ we have $0 \prec \mathbf{G}(\mathbf{y}+\mathbf{v})$.

In many engineering applications, some components of the state vector can be directly measured justifying the restriction on positivity of the matrix $\mathbf{C}$ elements. The requirement on Metzler property of the matrices $\mathbf{A}_{o}-\mathbf{L}_{o} \mathbf{C}, o \in\{m, M\}$ is always required for interval observation, see for instance [14]. This condition can be reformulated as an LMI:

$$
\begin{aligned}
& \mathbf{p}_{o}^{T} \mathbf{A}_{o}-\mathbf{w}_{o}^{T} \mathbf{C}<0, \quad \mathbf{p}_{o}^{T} \mathbf{A}_{o}-\mathbf{w}_{o}^{T} \mathbf{C}+\mathbf{r}_{o}^{T} \geq 0, \quad \mathbf{L}_{o}=\mathbf{p}_{o} \mathbf{w}_{o}^{T} \\
& \mathbf{p}_{o}>0, \quad \mathbf{r}_{o} \geq 0
\end{aligned}
$$

where $\mathbf{p}_{o} \in R^{n}, \mathbf{w}_{o} \in R^{p}$ and $\mathbf{r}_{o} \in R^{n}, o \in\{m, M\}$. It can be relaxed under a linear coordinate change [13], [23], i.e. it assumed that $\mathbf{A}_{o}-\mathbf{L}_{o} \mathbf{C}, o \in\{m, M\}$ are Hurwitz and it is required to find a nonsingular matrix $\mathbf{S} \in R^{n \times n}$ such that the matrices $\mathbf{S}^{-1}\left(\mathbf{A}_{o}-\mathbf{L}_{o} \mathbf{C}\right) \mathbf{S}, o \in\{m, M\}$ would be Hurwitz and Metzler, some tools to design of such a matrix $\mathbf{S}$ can be found in [13], [23].

Note that, since $\boldsymbol{\theta} \in \Theta$ there exist two vectors $\boldsymbol{\theta}_{m} \in R^{q}$ and $\boldsymbol{\theta}_{M} \in R^{q}$ such that $\boldsymbol{\theta}_{m} \leq \boldsymbol{\theta} \leq \boldsymbol{\theta}_{M}$ for 
all $\boldsymbol{\theta} \in \Theta$. The requirement of assumption 1 that the function $\mathbf{G}$ has positive values is essential for the proposed approach (a straightforward result reformulation is possible for the case of negative values of $\mathbf{G}$ ). The equations of the set adaptive observer have the form:

$$
\begin{gathered}
\dot{\zeta}_{o}=\mathbf{A}_{o} \zeta_{o}+\mathbf{B}_{o} \mathbf{u}+\phi\left(\mathbf{y}_{v}\right)+\mathbf{L}_{o}\left(\mathbf{y}_{v}-\mathbf{C} \zeta_{o}\right) ; \\
\dot{\boldsymbol{\Omega}}_{o}=\left[\mathbf{A}_{o}-\mathbf{L}_{o} \mathbf{C}\right] \boldsymbol{\Omega}_{o}-\mathbf{G}\left(\mathbf{y}_{v}\right) ; \\
\dot{\boldsymbol{\theta}}_{o}=-\boldsymbol{\Gamma}_{o} \boldsymbol{\Omega}_{o}^{T} \mathbf{C}^{T}\left(\mathbf{y}_{v}-\mathbf{C} \zeta_{o}+\mathbf{C} \boldsymbol{\Omega}_{o} \widehat{\boldsymbol{\theta}}_{o}\right)
\end{gathered}
$$

where the index $o \in\{m, M\}$ denotes the upper and lower interval bounds, $\zeta_{o} \in R^{n}$ is the interval estimate of $\mathbf{x}, \boldsymbol{\Omega}_{o} \in R^{n \times q}$ is an auxiliary filter variable and $\widehat{\boldsymbol{\theta}}_{o} \in R^{q}$ is the interval estimate of $\boldsymbol{\theta}$, the matrix $\boldsymbol{\Gamma}_{o}=\Gamma_{o}^{T}>0$ is a design parameter.

In set observer design the monotonicity property of observer equations plays an essential role. As it can be deduced from equations (6)-(8), the monotonicity of the first two subsystems (6), (7) is predefined by assumption 1. Monotonicity of the system (8), which defines dynamics of parameter estimator, may not be followed by the same property of (6), (7). Actually, it can be shown that under some conditions, the dynamics of (8) can be either cooperative or competitive, impacting the construction of the admissible set of $\boldsymbol{\theta}$. In the sequel, the competitive case is analyzed only.

Define $\varepsilon_{o}=\mathbf{x}-\zeta_{o}, o \in\{m, M\}$

Theorem 1. Let assumption 1 be satisfied, $\mathbf{x}(t) \in X, \mathbf{u}(t) \in U, \rho(t) \in \Upsilon, \boldsymbol{\theta} \in \Theta$ for all $t \geq 0$, and the signals $\mathbf{\Omega}_{o}^{T}(t) \mathbf{C}^{T}$ be $\left(\ell_{o}, \vartheta_{o}\right)-P E$ for some $\ell_{o}>0, \vartheta_{o}>0$ and all $o \in\{m, M\}$. Then:

(i) the solutions $\zeta_{o}(t), \mathbf{\Omega}_{o}(t)$ and $\widehat{\boldsymbol{\theta}}_{o}(t), o \in\{m, M\}$ of the system (6)-(8) are bounded for all $t \geq 0$ for a bounded noise $\mathbf{v}(t) \in V, t \geq 0$;

(ii) let $\mathbf{v}(t) \equiv 0, t \geq 0$ and $0 \prec \boldsymbol{\Gamma}_{o} \prec \overline{\boldsymbol{\Gamma}}, o \in\{m, M\}$ for some sufficiently small $\overline{\boldsymbol{\Gamma}}$, then

a. if $\boldsymbol{\Omega}_{o}(0)=0, o \in\{m, M\}, \boldsymbol{\varepsilon}_{m}(0) \geq 0, \boldsymbol{\varepsilon}_{M}(0) \leq 0, \hat{\boldsymbol{\theta}}_{M}(0)=\boldsymbol{\theta}_{m}, \widehat{\boldsymbol{\theta}}_{m}(0)=\boldsymbol{\theta}_{M}$ and there are $\mathbf{b}_{o}=-\lim _{T \rightarrow+\infty} T^{-1} \int_{0}^{T} \boldsymbol{\Omega}_{o}^{T}(t) \mathbf{C}^{T} \mathbf{C} \boldsymbol{\varepsilon}_{o}(t) d t, \mathbf{R}_{o}=\lim _{T \rightarrow+\infty} T^{-1} \int_{0}^{T} \boldsymbol{\Omega}_{o}^{T}(t) \mathbf{C}^{T} \mathbf{C} \boldsymbol{\Omega}_{o}(t) d t$, 
$o \in\{m, M\}$ such that $\boldsymbol{\theta}_{M}<\mathbf{R}_{m}^{-1} \mathbf{b}_{m}, \mathbf{R}_{M}^{-1} \mathbf{b}_{M}<\boldsymbol{\theta}_{m}$, then $\widehat{\boldsymbol{\theta}}_{M}(t) \leq \boldsymbol{\theta} \leq \hat{\boldsymbol{\theta}}_{m}(t), t \geq 0$.

b. if $\boldsymbol{\Omega}_{o}(0)=0, o \in\{m, M\}, \boldsymbol{\varepsilon}_{m}(0) \leq 0, \boldsymbol{\varepsilon}_{M}(0) \geq 0, \hat{\boldsymbol{\theta}}_{m}(0)=\boldsymbol{\theta}_{m}, \widehat{\boldsymbol{\theta}}_{M}(0)=\boldsymbol{\theta}_{M}$ and

$$
\boldsymbol{\theta}_{M}<\mathbf{R}_{M}^{-1} \mathbf{b}_{M}, \mathbf{R}_{m}^{-1} \mathbf{b}_{m}<\boldsymbol{\theta}_{m} \text {, then } \widehat{\boldsymbol{\theta}}_{m}(t) \leq \boldsymbol{\theta} \leq \widehat{\boldsymbol{\theta}}_{M}(t), t \geq 0
$$

Proof. Define $\varepsilon_{o}=\mathbf{x}-\zeta_{o}, \tilde{\boldsymbol{\theta}}_{o}=\boldsymbol{\theta}-\hat{\boldsymbol{\theta}}_{o}$ and $\boldsymbol{\delta}_{o}=\boldsymbol{\varepsilon}_{o}+\Omega_{o} \boldsymbol{\theta}$ for $o \in\{m, M\}$, then we obtain

$$
\begin{gathered}
\dot{\boldsymbol{\varepsilon}}_{o}=\left[\mathbf{A}_{o}-\mathbf{L}_{o} \mathbf{C}\right] \boldsymbol{\varepsilon}_{o}+\mathbf{G}\left(\mathbf{y}_{v}\right) \boldsymbol{\theta}+\mathbf{p}_{o}+\mathbf{d}_{v}, \\
\mathbf{p}_{o}=\left[\mathbf{A}(\rho(t))-\mathbf{A}_{o}\right] \mathbf{x}+\left[\mathbf{B}(\rho(t))-\mathbf{B}_{o}\right] \mathbf{u}, \mathbf{d}_{v}=\phi(\mathbf{y})-\phi\left(\mathbf{y}_{v}\right)+\left[\mathbf{G}(\mathbf{y})-\mathbf{G}\left(\mathbf{y}_{v}\right)\right] \boldsymbol{\theta}-\mathbf{L}_{o} \mathbf{v}, \\
\dot{\boldsymbol{\delta}}_{o}=\left[\mathbf{A}_{o}-\mathbf{L}_{o} \mathbf{C}\right] \boldsymbol{\delta}_{o}+\mathbf{p}_{o}+\mathbf{d}_{v}, \\
\dot{\tilde{\boldsymbol{\theta}}}_{o}=\boldsymbol{\Gamma}_{o} \mathbf{\Omega}_{o}^{T} \mathbf{C}^{T}\left(\mathbf{C} \boldsymbol{\delta}_{o}+\mathbf{v}-\mathbf{C} \boldsymbol{\Omega}_{o} \tilde{\boldsymbol{\theta}}_{o}\right) .
\end{gathered}
$$

The term $\mathbf{p}_{o}$ appears in (9), (10) due to the introduction of $\mathbf{A}_{o}, \mathbf{B}_{o}$ in (6)-(8) and $\mathbf{d}_{v}$ represents the noise influence. Owing to assumption 1, all solutions of the system (7) are bounded for $\mathbf{y} \in Y$, $\mathbf{v} \in V$, i.e. there exists $k_{\Omega, o}>0$ such that $\left|\Omega_{o}(t)\right| \leq k_{\Omega, o}$ for all $t \geq 0$. Then, $\left|\mathbf{d}_{v}\right| \leq\left[k_{\phi}+k_{G}|\boldsymbol{\theta}|+\left|\mathbf{L}_{o}\right|\right]|\mathbf{v}|$ and for $\boldsymbol{\theta} \in \Theta, \mathbf{v} \in V$ the signal $\mathbf{d}_{v}$ remains bounded. The signal $\mathbf{p}_{o}$ is bounded for any $\rho(t) \in \Upsilon, \mathbf{x}(t) \in X, \mathbf{u}(t) \in U$. Therefore, if assumption 1 is satisfied, then the solutions of (10) are bounded. In addition, if the signal $\mathbf{C}^{T} \mathbf{\Omega}_{o}^{T}(t)$ is persistently exciting, then from lemma 1 the solutions of system (11) remain bounded, and it follows that $\boldsymbol{\varepsilon}_{o}(t)=\boldsymbol{\delta}_{o}(t)-\boldsymbol{\Omega}_{o}(t) \boldsymbol{\theta}$ is bounded. Therefore, the first part of the theorem is proven.

Now, let $\mathbf{v}(t)=0$ for all $t \geq 0$, which implies $\mathbf{d}_{v}(t)=0, t \geq 0$. Since $0 \prec \mathbf{G}(\mathbf{y}+\mathbf{v})$ for all $\mathbf{y}(t) \in Y, \mathbf{v}(t) \in V, t \geq 0$, then monotonicity of the system (7) ensures that $\mathbf{\Omega}_{o}(t) \prec 0$ for all $t \geq 0$ and $o \in\{m, M\}$ for $\boldsymbol{\Omega}_{o}(0)=0$. In the equation (8) the gain matrix $\boldsymbol{\Gamma}_{o} \boldsymbol{\Omega}_{o}^{T}(t) \mathbf{C}^{T} \mathbf{C} \boldsymbol{\Omega}_{o}(t)$, $t \geq 0$ is positive semidefinite. Due to $0 \prec \mathbf{C}$ it is not negative elementwise for both $o \in\{m, M\}$. Then the system (8) is competitive [33]. The matrix coefficients $\Gamma_{o}, o \in\{m, M\}$ define the rate of changes for the variables $\widehat{\boldsymbol{\theta}}_{0}$. A modification of $\boldsymbol{\Gamma}_{o}, o \in\{m, M\}$ does not affect on behavior of the variables $\boldsymbol{\Omega}_{o}^{T}(t) \mathbf{C}^{T} \mathbf{C} \boldsymbol{\Omega}_{o}(t)$ and $\boldsymbol{\Omega}_{o}^{T}(t) \mathbf{C}^{T} \mathbf{C} \boldsymbol{\varepsilon}_{o}(t)$, which are defined in the equations (6), (7) decoupled from (8). If $\boldsymbol{\Gamma}_{o}, o \in\{m, M\}$ are chosen sufficiently small, then the variables $\hat{\boldsymbol{\theta}}_{0}(t)$ be- 
come "slowly-varying" in the system (3), (6)-(8) and the variables $\boldsymbol{\Omega}_{o}(t)$ and $\boldsymbol{\varepsilon}_{o}(t)$ are the "fast" ones. In such conditions, it is possible to apply averaging technique [6], [30]:

$$
\dot{\hat{\boldsymbol{\theta}}}_{o}(t)=\boldsymbol{\Gamma}_{o}\left[\mathbf{b}_{o}-\mathbf{R}_{o} \hat{\boldsymbol{\theta}}_{o}(t)\right]
$$

The matrices $\mathbf{R}_{o}, o \in\{m, M\}$ are positive definite due to PE condition $\left(\mathbf{R}_{o} \geq 0.5 \vartheta_{o} / \ell_{o} \mathbf{I}_{q}\right.$ according to lemma A1 from [10]). The system (12) is competitive and asymptotically stable. Its equilibrium is located not at the origin, but applying a linear coordinate shift we can ensure a realization of all required conditions of Proposition 1 (note that (11) is a linear time varying system, therefore all continuity and differentiability requirements imposed in Proposition 1 on $\mathbf{f}$ and $\overline{\mathbf{f}}$ are valid). The solutions of the system (12) asymptotically converge to the equilibrium $\hat{\boldsymbol{\theta}}_{o}^{\infty}=\mathbf{R}_{o}^{-1} \mathbf{b}_{o}$. Since $\widehat{\boldsymbol{\theta}}_{m}(0)=\boldsymbol{\theta}_{M}<\mathbf{R}_{m}^{-1} \mathbf{b}_{m}$ and $\mathbf{R}_{M}^{-1} \mathbf{b}_{M}<\boldsymbol{\theta}_{m}=\widehat{\boldsymbol{\theta}}_{M}(0)$, then using relations between solutions of a stable averaged system and the original one we get that $\lim _{t \rightarrow+\infty} \widehat{\boldsymbol{\theta}}_{m}(t) \geq \boldsymbol{\theta}_{M}, \lim _{t \rightarrow+\infty} \widehat{\boldsymbol{\theta}}_{M}(t) \leq \boldsymbol{\theta}_{m}$ (see Proposition 1 , if at the equilibrium of the averaged system a strict inequality holds, then there exists $\varepsilon>0$ small enough (the matrix $\overline{\boldsymbol{\Gamma}}$ ) such that the inequality is satisfied for solutions of the original system). These facts imply that $\tilde{\boldsymbol{\theta}}_{m}(t) \leq 0, \tilde{\boldsymbol{\theta}}_{M}(t) \geq 0$ for all $t \geq 0$. The part (ii).a of the theorem has been established. The part (ii).b can be proven in the same way.

To ensure the constraints on initial conditions $\boldsymbol{\varepsilon}_{m}(0), \boldsymbol{\varepsilon}_{M}(0)$ the values $\mathbf{x}_{m}, \mathbf{x}_{M}$ can be used.

Remark 1. The conditions dealing with computation of $\mathbf{R}_{o}$ and $\mathbf{b}_{o}, o \in\{m, M\}$ are the most restrictive in Theorem 1, they can be verified after the observer (6)-(8) run. Instead the values $\widehat{\boldsymbol{\theta}}_{o}^{\infty}=\mathbf{R}_{o}^{-1} \mathbf{b}_{o}, o \in\{m, M\}$ can be evaluated and compared on-line with $\boldsymbol{\theta}_{m}$ and $\boldsymbol{\theta}_{M}$, i.e. the estimates

$$
\widehat{\mathbf{b}}_{o}(t)=-t^{-1} \int_{0}^{t} \boldsymbol{\Omega}_{o}^{T}(\tau) \mathbf{C}^{T} \mathbf{C} \boldsymbol{\varepsilon}_{o}(\tau) d \tau, \widehat{\mathbf{R}}_{o}(t)=t^{-1} \int_{0}^{t} \boldsymbol{\Omega}_{o}^{T}(\tau) \mathbf{C}^{T} \mathbf{C} \boldsymbol{\Omega}_{o}(\tau) d \tau
$$

are well defined for all finite $t \geq \ell_{o}, o \in\{m, M\}$ (by lemma A1 from [10], the matrix $\widehat{\mathbf{R}}_{o}(t)$ is not singular for $\left.t \geq \ell_{o}\right)$ and the variable $\overline{\boldsymbol{\theta}}_{o}^{\infty}(t)=\hat{\mathbf{R}}_{o}^{-1}(t) \hat{\mathbf{b}}_{o}(t)$ can be used for $\hat{\boldsymbol{\theta}}_{o}^{\infty}$ evaluation. There- 
fore, as far as the restrictions $\overline{\boldsymbol{\theta}}_{o}^{\infty}(t) \approx \widehat{\boldsymbol{\theta}}_{o}^{\infty}, o \in\{m, M\}$ required in theorem 1 are satisfied, the observers generate reliable interval estimates for the vector $\boldsymbol{\theta}$. Under (13), the obtained solution becomes approximate.

Remark 2 . Note that PE property of the signals $\boldsymbol{\Omega}_{o}^{T}(t) \mathbf{C}^{T}, o \in\{m, M\}$ can also be checked on-line by computing the integrals $\int_{t}^{t+\ell_{o}} \boldsymbol{\Omega}_{o}^{T}(\tau) \mathbf{C}^{T} \mathbf{C} \boldsymbol{\Omega}_{o}(\tau) d \tau, o \in\{m, M\}$ for some $\ell_{o}>0$ for all $t \geq 0$. As far as these integrals are nonsingular, the PE property holds. According to lemma A1 in [10], non-singularity of these integrals are equivalent to the same property of $t^{-1} \int_{0}^{t} \mathbf{\Omega}_{o}^{T}(\tau) \mathbf{C}^{T} \mathbf{C} \boldsymbol{\Omega}_{o}(\tau) d \tau$, which coincides with $\widehat{\mathbf{R}}_{o}(t)$ from (13).

Remark 3. If the functions $\mathbf{C} \boldsymbol{\Omega}_{o}(t)$ and $\mathbf{C} \boldsymbol{\varepsilon}_{o}(t)$ are $T$-periodical, then the limits can be dropped in the definitions of $\mathbf{h}_{o}$ and $\mathbf{R}_{o}, o \in\{m, M\}$ in theorem 1 formulation [30]. In this case, on-line verification of the conditions for $\mathbf{R}_{o}^{-1} \mathbf{b}_{o}$ via (13) becomes trivial.

Remark 4. It is important to note that the conditions of assumption 1 deal with the constant matrices $\mathbf{A}_{o}$, while an application of the conventional approaches [10], [11], [34], [37], [39], [40] to the system (3) needs the measurements of the vector of scheduling parameters $\rho(t)$ and the stability conditions involving the time-varying matrix $\mathbf{A}[\rho(t)]$. This fact justifies the set observers application when the conventional observers cannot be realized.

Remark 5. From another side, if $\Upsilon=\varnothing$, then the observers (6)-(8) for $o=m$ and $o=M$ become identical and equal to a conventional adaptive observer from [10], [11], [34], [37], [39], [40]. Therefore, in this case the observers (6)-(8) have to ensure the exact estimation of the vector of uncertain parameters $\boldsymbol{\theta}$. Inversely, the width of the estimated interval is proportional to the uncertainty of (3) represented by the vector of the scheduling parameters $\rho$ (and the size of $\Upsilon$ ).

An advantage of the designed solution is that exponential complexity, which is usual for setmembership parameter estimation [16], [18], [27] with the branch-and-bound approach, is avoided. 


\section{State set observer}

From Theorem 1 we know that (6)-(8) generates interval estimates of the vector of unknown parameters: $\widehat{\boldsymbol{\theta}}_{O_{m}} \leq \boldsymbol{\theta} \leq \widehat{\boldsymbol{\theta}}_{O_{M}}, O_{m} \in\{m, M\}, O_{M}=\{m, M\} \backslash O_{m}$. Consider the following observers

$$
\dot{\boldsymbol{\xi}}_{o}=\mathbf{A}_{o} \boldsymbol{\xi}_{o}+\mathbf{B}_{o} \mathbf{u}+\phi\left(\mathbf{y}_{v}\right)+\mathbf{G}\left(\mathbf{y}_{v}\right) \widehat{\boldsymbol{\theta}}_{O_{o}}+\mathbf{L}_{o}\left(\mathbf{y}_{v}-\mathbf{C} \boldsymbol{\xi}_{o}\right), o, O_{o} \in\{m, M\},
$$

where $\hat{\boldsymbol{\theta}}_{O_{o}}, O_{o} \in\{m, M\}$ are generated by (8) and $\xi_{o} \in R^{n}, o \in\{m, M\}$ are the state estimates. The equation (14) partly repeats (6), however, the state $\zeta_{o}, o \in\{m, M\}$ of the system (6) cannot be used for the state $\mathbf{x}$ interval estimation since one of the inequalities $\widehat{\boldsymbol{\theta}}_{m}<\widehat{\boldsymbol{\theta}}_{M}$ or $\widehat{\boldsymbol{\theta}}_{M}<\widehat{\boldsymbol{\theta}}_{m}$ holds depending on the auxiliary conditions. This is why an additional index $O_{o}$ is introduced in (14).

Theorem 2. Let assumption 1 be satisfied, $\mathbf{x}(t) \in X, \mathbf{u}(t) \in U, \rho(t) \in \Upsilon, \boldsymbol{\theta} \in \Theta$ for all $t \geq 0$, and the signals $\mathbf{\Omega}_{o}^{T}(t) \mathbf{C}^{T}$ be $\left(\ell_{o}, \vartheta_{o}\right)-P E$ for some $\ell_{o}>0, \vartheta_{o}>0, o \in\{m, M\}$. Then

(i) the solutions $\xi_{o}(t), \zeta_{o}(t), \boldsymbol{\Omega}_{o}(t)$ and $\hat{\boldsymbol{\theta}}_{o}(t), o \in\{m, M\}$ of the system (6)-(8), (14) are bounded for all $t \geq 0$ for a bounded noise $\mathbf{v}(t) \in V, t \geq 0$;

(ii) let $\mathbf{v}(t) \equiv 0, \mathbf{x}(t) \geq 0, \mathbf{u}(t) \geq 0$ for all $t \geq 0$ and theorem 1, part (ii) conditions be satisfied indicating that $\widehat{\boldsymbol{\theta}}_{o}(t) \leq \boldsymbol{\theta} \leq \widehat{\boldsymbol{\theta}}_{O}(t), o, O \in\{m, M\}, t \geq 0$, then $\xi_{m}(t) \leq \mathbf{x}(t) \leq \xi_{M}(t)$ for all $t \geq 0$ provided that $\xi_{m}(0)=\mathbf{x}_{m}, \xi_{M}(0)=\mathbf{x}_{M}$ and $O_{m}=o, O_{M}=O$ in (14);

(iii) let $\mathbf{v}(t) \equiv 0, \mathbf{x}(t) \leq 0, \mathbf{u}(t) \leq 0$ for all $t \geq 0$ and theorem 1, part (ii) conditions be satisfied indicating that $\widehat{\boldsymbol{\theta}}_{o}(t) \leq \boldsymbol{\theta} \leq \widehat{\boldsymbol{\theta}}_{O}(t), o, O \in\{m, M\}, t \geq 0$, then $\xi_{M}(t) \leq \mathbf{x}(t) \leq \boldsymbol{\xi}_{m}(t)$ for all $t \geq 0$ provided that $\xi_{M}(0)=\mathbf{x}_{m}, \xi_{m}(0)=\mathbf{x}_{M}$ and $O_{m}=O, O_{M}=o$ in (14).

Proof. Consider the estimation errors $\mathbf{e}_{o}=\mathbf{x}-\boldsymbol{\xi}_{o}, o, O_{o} \in\{m, M\}$,

$$
\dot{\mathbf{e}}_{o}=\left[\mathbf{A}_{o}-\mathbf{L}_{o} \mathbf{C}\right] \mathbf{e}_{o}+\mathbf{G}\left(\mathbf{y}_{v}\right)\left[\boldsymbol{\theta}-\widehat{\boldsymbol{\theta}}_{O_{o}}\right]+\mathbf{d}_{v}+\mathbf{p}_{o} .
$$

Since all conditions of theorem 1, part (i) are satisfied, then the solutions $\zeta_{o}(t), \boldsymbol{\Omega}_{o}(t)$ and $\hat{\boldsymbol{\theta}}_{o}(t)$ are bounded for both $o \in\{m, M\}$. While $\mathbf{x}(t) \in X, \mathbf{u}(t) \in U, \mathbf{v}(t) \in V, \rho(t) \in \Upsilon$ and $\boldsymbol{\theta} \in \Theta$ the signals $\mathbf{p}_{o}(t), o \in\{m, M\}$ and $\mathbf{d}_{v}(t)$ remain bounded, and (15) is an asymptotically stable coop- 
erative linear system with a bounded input $\mathbf{G}\left(\mathbf{y}_{v}\right)\left[\boldsymbol{\theta}-\widehat{\boldsymbol{\theta}}_{O_{o}}\right]+\mathbf{d}_{v}+\mathbf{p}_{o}$, which implies boundedness of $\xi_{o}(t), o \in\{m, M\}$. The part (i) has been proven. The parts (ii) and (iii) can be proven similarly.

To apply the observer (6)-(8), (14) it is necessary to check the conditions of assumption 1 and verify boundedness of $\mathbf{x}(t) \in X, \mathbf{u}(t) \in U, \mathbf{v}(t) \in V, \rho(t) \in \Upsilon$ and $\boldsymbol{\theta} \in \Theta$. Next, the PE property as well as the integral constraints from theorem 1 can be verified on-line using (13).

\section{Fault detection}

The main idea of model-based fault detection and diagnosis is to check whether the behavior of a plant is consistent with its fault-free model. Many model-based approaches use the estimation of some relevant internal or observed variables to produce fault-indicating signals (residuals), see [4], [8], [12], [26], [29] and [9] for a recent survey. The set observers (6)-(8), (14) provide the estimates on the interval of current values of unmeasured states and uncertain parameters, this information can be sufficient for fault detection and isolation (the estimate of the true value is not required).

In this section we assume that in the system (3) the faults appearance is modeled by the vector $\boldsymbol{\theta}$ (the absence of faults corresponds to the case $\boldsymbol{\theta}=0$ ). The problem is to detect a significant change of the vector $\boldsymbol{\theta}$ value as fast as possible.

To solve this problem, in [28] it is proposed to use the following set observers:

$$
\dot{\zeta}_{o}=\mathbf{A}_{o} \zeta_{o}+\mathbf{B}_{o} \mathbf{u}+\phi\left(\mathbf{y}_{v}\right)+\mathbf{L}_{o}\left(\mathbf{y}_{v}-\mathbf{C} \zeta_{o}\right), o \in\{m, M\},
$$

which coincide with (6). The observers (6) estimate the interval of $\mathbf{x}$ for the nominal fault-free case $\boldsymbol{\theta}=0$. In this case $\hat{\mathbf{y}}_{m}(t) \leq \mathbf{y}(t) \leq \hat{\mathbf{y}}_{M}(t)$ for all $t \geq 0, \hat{\mathbf{y}}_{m}=\mathbf{C} \zeta_{m}, \hat{\mathbf{y}}_{M}=\mathbf{C} \zeta_{M}$. If these constraints are not satisfied, this would indicate that a fault is developing [5], [28]:

$$
S(t)=s_{1}(t) \vee \ldots \vee s_{p}(t), s_{i}(t)=\left\{\begin{array}{l}
0 \text { if } \hat{y}_{m, i}(t) \leq y_{i}(t) \leq \hat{y}_{M, i}(t), i=\overline{1, p}, \\
1 \text { otherwise, }
\end{array}\right.
$$

then $S(t)=0$ in the nominal case and $S(t)=1$ if a fault is detected (the symbol $\vee$ is used for the "logic or"). A method for estimation of the smallest detectable fault for (6) is discussed in [5], [28].

What new can be added to this procedure with application of (6)-(8) and (14)? Firstly, note that 
(6) is incorporated in the set adaptive observer, therefore the indicator (16) can be still verified. Secondly, the observers (6)-(8) provide the interval estimation for the fault vector $\boldsymbol{\theta}$ directly, which allows us to generate an additional fault indicating signal as follows:

$$
D(t)=d_{1}(t) \vee \ldots \vee d_{q}(t), d_{j}(t)=\left\{\begin{array}{l}
0 \text { if } \widehat{\theta}_{m, j}(t) \leq 0 \leq \widehat{\theta}_{M, i}(t), j=\overline{1, q} . \\
1 \text { otherwise, }
\end{array}\right.
$$

Under the conditions of theorem 1, a deviation of the signal (17) from zero indicates a fault appearance, while the variables $\widehat{\boldsymbol{\theta}}_{m}, \widehat{\boldsymbol{\theta}}_{M}$ evaluate the admissible interval of the fault $\boldsymbol{\theta}$ (that could be used in the fault isolation procedure). And finally, the observer (14) estimates the state $\mathbf{x}$ values taking into account the interval $\left[\widehat{\boldsymbol{\theta}}_{m}, \widehat{\boldsymbol{\theta}}_{M}\right]$, i.e. the condition $\xi_{m}(t) \leq \mathbf{x}(t) \leq \xi_{M}(t)$ approves the interval $\left[\widehat{\boldsymbol{\theta}}_{m}, \widehat{\boldsymbol{\theta}}_{M}\right]$. Then a third indicating signal can be defined as follows for $\widehat{\boldsymbol{\Psi}}_{m}=\mathbf{C} \boldsymbol{\xi}_{m}, \widehat{\boldsymbol{\Psi}}_{M}=\mathbf{C} \boldsymbol{\xi}_{M}$ :

$$
Z(t)=z_{1}(t) \vee \ldots \vee z_{p}(t), z_{i}(t)=\left\{\begin{array}{l}
0 \text { if } \widehat{\psi}_{m, i}(t) \leq y_{i}(t) \leq \widehat{\psi}_{M, i}(t), i=\overline{1, p} \\
1 \text { otherwise }
\end{array}\right.
$$

The case $Z(t)=0$ corresponds to the situation when $\widehat{\boldsymbol{\theta}}_{m} \leq \boldsymbol{\theta} \leq \widehat{\boldsymbol{\theta}}_{M}$ and $\boldsymbol{\xi}_{m}(t) \leq \mathbf{x}(t) \leq \boldsymbol{\xi}_{M}(t)$, while $Z(t)=1$ indicates the opposite status, i.e. the model (3) is not representative at all for the deviation appeared in the system (with or without faults). Therefore, the proposed approach consists in a simultaneous verification of the test signals (16)-(18). That gives a deeper insight into the situation for fault detection and isolation than the approach based on the state estimation (16) only.

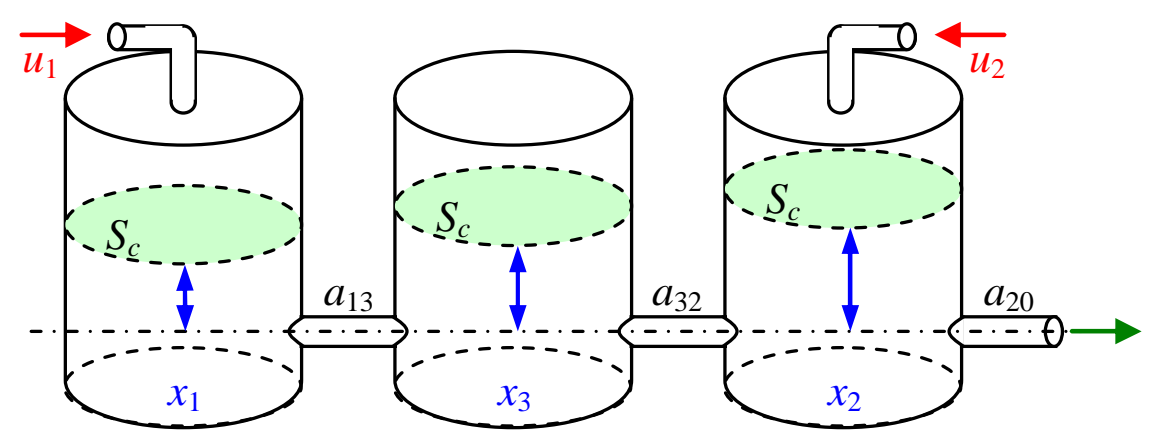

Fig. 1. The structure scheme of the system (19).

Example. As in [19], [28], [36], [41] consider the three-tank-system model: 


$$
\begin{gathered}
S_{c} \dot{x}_{1}=-a_{13} \rho\left(x_{1}-x_{3}\right)+u_{1}+\theta_{1}, \rho(x)=\operatorname{sign}(x) \sqrt{|x|} \\
S_{c} \dot{x}_{2}=-a_{32} \rho\left(x_{3}-x_{2}\right)-a_{20} \rho\left(x_{2}\right)+u_{2}+\theta_{2} ; \\
S_{c} \dot{x}_{3}=a_{13} \rho\left(x_{1}-x_{3}\right)-a_{32} \rho\left(x_{3}-x_{2}\right), \boldsymbol{\theta}=\left[\theta_{1} \theta_{2}\right]^{T},
\end{gathered}
$$

where the variables $x_{i}>0, i=\overline{1,3}$ denote the liquids levels in the corresponding tanks, $\mathbf{x}=\left[x_{1} \ldots x_{3}\right]^{T} ; u_{j}, j=1,2$ are pump flows attached to the tanks 1 and $2, \mathbf{u}=\left[u_{1} u_{2}\right]^{T} ; S_{c}$ is the cross section area of the tanks; the tanks are connected via the pipes with outflow coefficients $a_{13}=a_{32}$ and $a_{20}$ is the nominal outflow coefficient, $\mathbf{a}=\left[\begin{array}{lll}a_{13} & a_{32} & a_{20}\end{array}\right]^{T}$. The structure scheme of this system is given in Fig. 1. Actuator faults in the tanks 1 and 2 are modeled by $\theta_{1}$ and $\theta_{2}$.

It is required to design a fault detection system for the model (19). As in [28] we assume that only the variables $x_{1}$ and $x_{2}$ are available for measurements and the nominal values of the model (19) parameters $\left(a_{13}, a_{32}, a_{20}\right.$ and $\left.S_{c}\right)$ are given. The parameter $S_{c}$ is typically known and is not changing during normal operation. The domain $\mathbf{x}_{m} \leq \mathbf{x}(t) \leq \mathbf{x}_{M}$ for all $t \geq 0$ is given.

To apply the approach proposed here we need to transform the system (19) (which is in the form of the system (1)) to the form of (3). Denote by $\rho(x) / x=\lambda(x)=|x|^{-0.5}$, then the model (19) can be rewritten as follows:

$$
\begin{gathered}
\dot{\mathbf{x}}=\mathbf{A}(\mathbf{x}, \mathbf{a}) \mathbf{x}+\mathbf{B} \mathbf{u}+S_{c}^{-1} \boldsymbol{\theta}, \mathbf{B}=S_{c}^{-1}\left[\begin{array}{lll}
1 & 0 & 0 \\
0 & 1 & 0
\end{array}\right]^{T} \\
\mathbf{A}(\mathbf{x}, \mathbf{a})=S_{c}^{-1}\left[\begin{array}{ccc}
-a_{13} \lambda\left(x_{1}-x_{3}\right) & a_{13} \lambda\left(x_{1}-x_{3}\right) \\
0 & 0 & a_{32} \lambda\left(x_{3}-x_{2}\right) \\
a_{13} \lambda\left(x_{1}-x_{3}\right) & a_{32} \lambda\left(x_{3}-x_{2}\right)-a_{20} \lambda\left(x_{2}\right) & -a_{32} \lambda\left(x_{3}-x_{2}\right)-a_{13} \lambda\left(x_{1}-x_{3}\right)
\end{array}\right],
\end{gathered}
$$

that is similar to (3). From (20) we get

$$
\begin{gathered}
\mathbf{C}=\left[\begin{array}{lll}
1 & 0 & 0 \\
0 & 1 & 0
\end{array}\right], \mathbf{G}=\mathbf{B}, \mathbf{L}_{m}=\mathbf{L}_{M}=\ell\left[\begin{array}{lll}
1 & 0 & 0 \\
0 & 1 & 0
\end{array}\right]^{T}, \ell>0, \\
\mathbf{A}_{m}(\mathbf{y})=S_{c}^{-1}\left[\begin{array}{ccc}
-a_{13} \lambda\left(y_{1}-x_{M, 3}\right) & 0 & a_{13} \lambda\left(y_{1}-x_{m, 3}\right) \\
0 & -a_{32} \lambda\left(x_{m, 3}-y_{2}\right)-a_{20} \lambda\left(y_{2}\right) & a_{32} \lambda\left(x_{M, 3}-y_{2}\right) \\
a_{13} \lambda\left(y_{1}-x_{m, 3}\right) & a_{32} \lambda\left(x_{M, 3}-y_{2}\right) & -a_{32} \lambda\left(x_{m, 3}-y_{2}\right)-a_{13} \lambda\left(y_{1}-x_{M, 3}\right)
\end{array}\right], \\
\mathbf{A}_{M}(\mathbf{y})=S_{c}^{-1}\left[\begin{array}{ccc}
-a_{13} \lambda\left(y_{1}-x_{m, 3}\right) & 0 & a_{13} \lambda\left(y_{1}-x_{M, 3}\right) \\
0 & -a_{32} \lambda\left(x_{M, 3}-y_{2}\right)-a_{20} \lambda\left(y_{2}\right) & a_{32} \lambda\left(x_{m, 3}-y_{2}\right) \\
a_{13} \lambda\left(y_{1}-x_{M, 3}\right) & a_{32} \lambda\left(x_{m, 3}-y_{2}\right) & -a_{32} \lambda\left(x_{M, 3}-y_{2}\right)-a_{13} \lambda\left(y_{1}-x_{m, 3}\right)
\end{array}\right] .
\end{gathered}
$$


Clearly, the matrices $\mathbf{A}_{m}$ and $\mathbf{A}_{M}$ are Metzler and for the chosen gains $\mathbf{L}_{m}, \mathbf{L}_{M}$ the conditions of assumption 1 are satisfied. The control algorithms are chosen as follows

$$
u_{1}\left(t, y_{1}\right)=v\left(-k \rho\left(y_{1}-y_{r, 1}(t)\right)\right), u_{2}\left(t, y_{2}\right)=v\left(-k \rho\left(y_{2}-y_{r, 2}(t)\right)+a_{20} \rho\left(y_{2}\right)\right) \text {, }
$$

where $\mathbf{y}_{r}(t)=\left[y_{r, 1}(t) y_{r, 2}(t)\right]^{T}$ is the reference signal to be tracked by $x_{1}$ and $x_{2} ; k>0$ is the control gain, $v(u)=\left\{\begin{array}{ll}u & \text { if } u>0 ; \\ 0 & \text { otherwise, }\end{array}\right.$. The following values of parameters are used for simulation:

$$
\begin{gathered}
a_{13}=a_{32}=1.329 \times 10^{-4}, a_{20}=1.772 \times 10^{-4}, S_{c}=0.0154, k=1.329 \times 10^{-3}, \ell=3, \\
\mathbf{x}_{m}=\left[\begin{array}{lll}
0.44 & 0.04 & 0.24
\end{array}\right]^{T}, \mathbf{x}_{M}=\left[\begin{array}{lll}
0.56 & 0.16 & 0.36
\end{array}\right]^{T}, T=200, \mathbf{x}(0)=0.5\left(\mathbf{x}_{m}+\mathbf{x}_{M}\right), \\
\mathbf{y}_{r}(t)=\left[\begin{array}{lll}
0.5(1+0.07 \mu(t)) & 0.1(1+0.5 \mu(t))
\end{array}\right]^{T}, \mu(t)=\left\{\begin{array}{l}
0 \text { if } t \bmod T \leq T / 2 ; \\
1 \text { otherwise }
\end{array}\right.
\end{gathered}
$$

a.
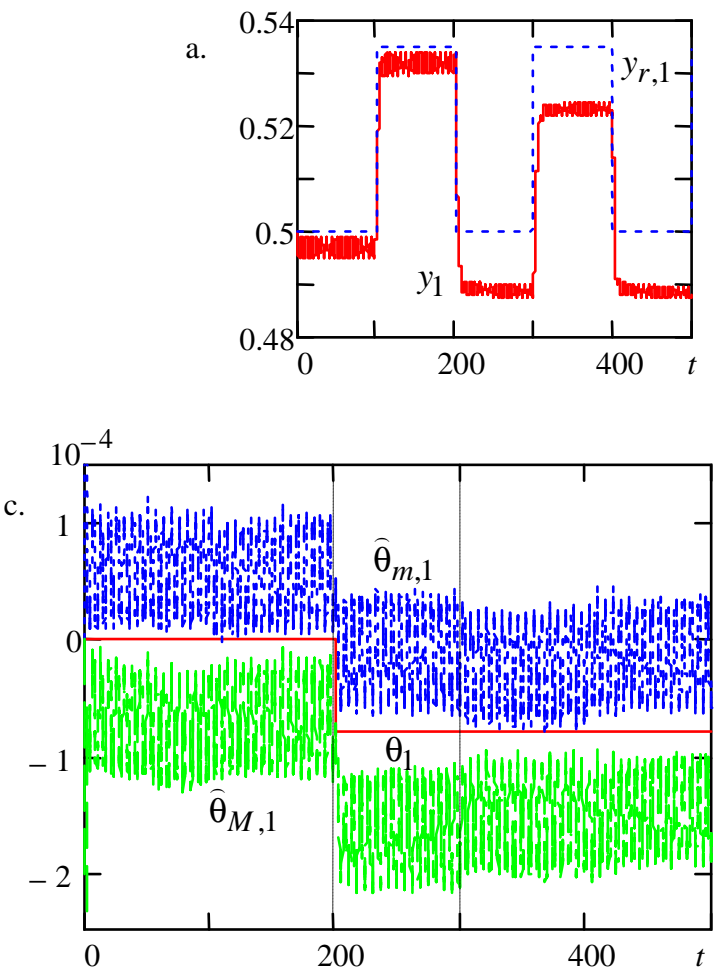

e.

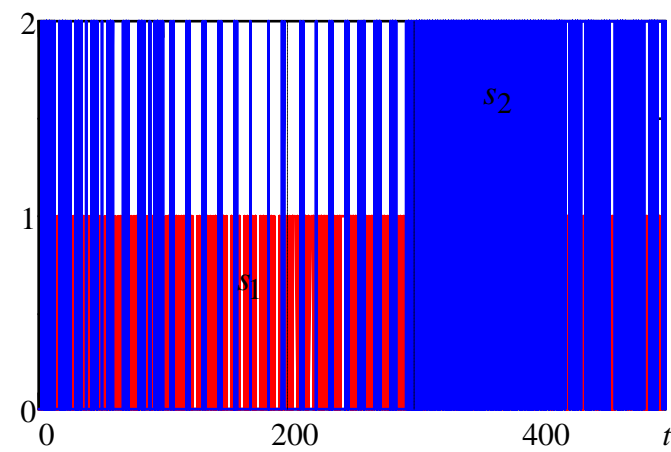

b.

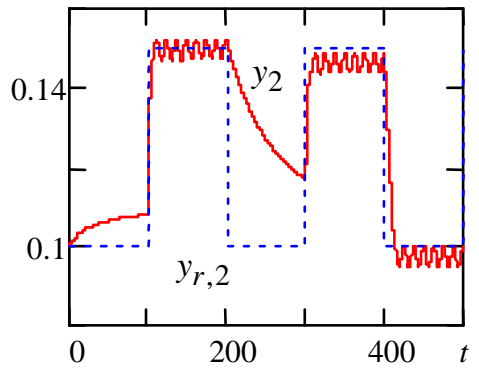

d.
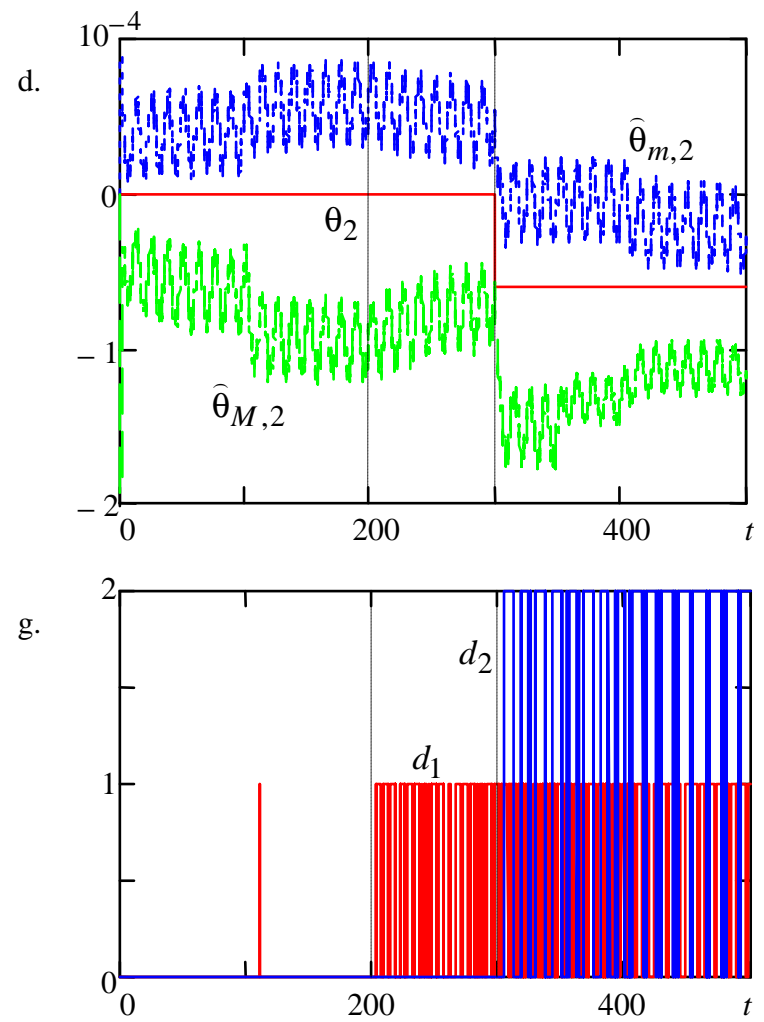

Fig. 2. Simulation results with noise: output $\mathbf{y}$ and its reference $\mathbf{y}_{d}((\mathrm{a}),(\mathrm{b}))$; $\widehat{\boldsymbol{\theta}}_{o}$ for $o \in\{m, M\}$ $((\mathrm{c}),(\mathrm{d}))$; fault indicating signals $\mathbf{s}$ and $\mathbf{d}((\mathrm{e}),(\mathrm{g}))$. 
For simulation, it is assumed that two faults modeled by $\theta_{1}=8 \times 10^{-5}$ and $\theta_{2}=6 \times 10^{-5}$ appear at the time instants $t_{1}=200 \mathrm{sec}$ and $t_{2}=300 \mathrm{sec}$ respectively. The corresponding trajectories are shown in Fig. 2 for the case of a stochastic noise presence $|\mathbf{v}(t)| \leq 4.5 \times 10^{-3}$ (the outputs are plotted in Fig. 2,a and b, the graphics of $\boldsymbol{\theta}, \widehat{\boldsymbol{\theta}}_{m}, \widehat{\boldsymbol{\theta}}_{M}$ are presented in Fig. 2,c and d, the scaled indicating signals $s_{i}, d_{i}, i=1,2$ are shown in Fig. 2,e and $\mathrm{g}$, the signals $z_{i}$ are not presented since they are zero during all time of the simulation). The both parametric estimates quickly follow the faults, the fault detection delays are $0.35 \mathrm{sec}$ and $0.45 \mathrm{sec}$ respectively based on the signals $d_{1}$ and $d_{2}$ only. As it can be seen from the figure, the faults indicating signals $d_{i}, i=1,2$ are less sensitive to the measurement noise than $s_{i}, i=1,2$. In this example, based on $d_{i}, i=1,2$ it is possible to detect faults even in the case of rather noisy measurements.

Generally speaking, the numerical experiments show that the proposed approach is suitable in applications where reliable and robust detection of abnormal situations is of primary interest: some characteristic parameters deviate from their normal regime during on-line operation.

\section{Conclusion}

The basic problem studied by this paper is a set adaptive observer design for joint parameter and state estimation for nonlinear/LPV systems with not measured vector of scheduling parameters. The exponential complexity, usual for set-membership parameter estimation in nonlinear continuoustime systems, is avoided. The complexity of the proposed observer is similar to the Kalman filter and the dimension of the adaptive set observer equations increases proportionally to the parameter $\boldsymbol{\theta}$ and to the state $\mathbf{x}$ dimensions (the full dimension is $2(2 n+n \times q+q))$. This feature makes possible applications of observers for high dimensional uncertain systems. The accuracy of the proposed approach is characterized by the interval length, which is proportional to the "size" of uncertainty given in the set $\Upsilon$.

Finally, the set adaptive observers have been applied to solve the problem of parametric fault de- 
tection. The simulation results confirm fault detection ability and robustness of the proposed set adaptive observers.

\section{References}

[1] Anderson B.D.O. Exponential stability of linear equations arising in adaptive identification. IEEE Trans. Automat. Control, 22, 1977, pp. 83-88.

[2] Bernard O., Gouzé J.L. Closed loop observers bundle for uncertain biotechnological models. $J$. Process Control, 14, 2004, pp. 765-774.

[3] Besançon G. (Ed.) Nonlinear observers and applications. Lecture Notes in Control and Inforamtion Science, v. 363, Springer Verlag: Berlin, 2007.

[4] Blanke M., Kinnaert M., Lunze J., Staroswiecki M. Diagnosis and Fault Tolerant Control. Springer-Verlag: Berlin, 2nd ed, 2006.

[5] Blesa J., Puig V., Bolea Y. Fault detection using interval LPV models in an open-flow canal. Control Engineering Practice, 18(5), 2010, pp. 460-470.

[6] Bogoliubov N.N., Mitropolskii Yu.A. Asymptotic methods in the theory of nonlinear oscillations. New York: Gordon and Breach, 1961.

[7] Bokor, J., Balas G. Detection Filter Design for LPV Systems - a Geometric Approach. Automatica, 40, 2004, pp. 511-518.

[8] Chen J., Patton R.J. Robust model-based fault diagnosis for dynamic systems. Kluwer Academic Publishers, 1999.

[9] Ding S.X. Model-based Fault Diagnosis Techniques. Design Schemes, Algorithms, and Tools. Springer, Heidelberg, Berlin, 2008.

[10] Efimov D. Dynamical adaptive synchronization. Int. J. Adaptive Control and Signal Processing, 20(9), 2006, pp. 491-507.

[11] Farza M., M'Saad M., Maatoug T., Kamoun M. Adaptive observers for nonlinearly parameterized class of nonlinear systems. Automatica, 45(10), 2009, pp. 2292-2299.

[12] Ferrari R., Parisini T., Polycarpou M.M. Distributed Fault Diagnosis with Overlapping Decompositions: an Adaptive Approximation Approach. IEEE Trans. on Automatic Control, 54(4), 2009, pp. 794-799.

[13] Raïssi T., Efimov D., Zolghadri A. Interval state estimation for a class of nonlinear systems. IEEE Trans. Automatic Control, 57(1), 2012, pp. 260-265.

[14] Gouzé J.L., Rapaport A., Hadj-Sadok M.Z. Interval observers for uncertain biological systems. Ecological Modeling, 133, 2000, pp. 46-56.

[15] Jaulin L. Nonlinear bounded-error state estimation of continuous time systems. Automatica, 
38(2), 2002, pp. 1079-1082.

[16] Jaulin L., Walter E. Set inversion via interval analysis for nonlinear bounded-error estimation. Automatica, 29(4), 1993, pp. 1053-1064.

[17] Javad M., Carsten S.W. (Eds.) Control of Linear Parameter Varying Systems with Applications. Springer, 2012.

[18] Johnson T., Tucker W. Rigorous parameter reconstruction for differential equations with noisy data. Automatica, 44(9), 2008, pp. 2422-2426.

[19] Join C., Sira-Ramirez H., Fliess M. Control of an uncertain three tank system via on-line parameter identification and fault detection. Proc. 16th IFAC World Congress, Prague, 2005.

[20] Kieffer M., Walter E. Guaranteed nonlinear state estimator for cooperative systems. Numerical Algorithms, 37, 2004, pp. 187-198.

[21] Lee L.H. Identification and Robust Control of Linear Parameter-Varying Systems. PhD thesis, University of California at Berkeley, Berkeley, California, 1997.

[22] Marcos A., Balas J. Development of linear-parameter-varying models for aircraft. J. Guidance, Control, Dynamics, 27(2), 2004.

[23] Mazenc F., Bernard O. Interval observers for linear time-invariant systems with disturbances, Automatica, 47(1), 2011, pp. 140-147.

[24] Müller M. Überdas fundamental theorem in der theorie der gewöhnlichen differentialgleichungen. Math. Z, 26, 1920, pp. 619-645.

[25] Nijmeijer H., Fossen T.I. New Directions in Nonlinear Observer Design. London: SpringerVerlag, 1999.

[26] Puig V. Fault diagnosis and fault tolerant control using set-membership approaches: application to real case studies. Int. J. Appl. Math. Comput. Sci., 2010, 20(4), pp. 619-635

[27] Raïssi T., Ramdani N., Candau Y. Set membership state and parameter estimation for systems described by nonlilear differential equations. Automatica, 40, 2004, pp. 1771-1777.

[28] Raïssi T., Videau G., Zolghadri A. Interval observers design for consistency checks of nonlinear continuous-time systems. Automatica, 46(3), 2010, pp. 518-527.

[29] Rosa P., Silvestre C., Shamma J.S., and Athans M. Fault detection and isolation of LTV systems using set-valued observers. Proc. 49th IEEE Conference on Decision and Control, December 2010, Atlanta, pp. 768-773.

[30] Sanders J., Verhulst F., Murdock J. Averaging Methods in Nonlinear Dynamical Systems. NY: Springer, 2007.

[31] dos Santos P.L., Perdicoúlis T.P.A., Novara C., Ramos J.A., Rivera D.E. (Eds.) Linear Parameter-Varying System Identification : New Developments and Trends. Advanced Series in Electrical and Computer Engineering, 14, World Scientific, 2011. 
[32] Shamma J., Cloutier J. Gain-scheduled missile autopilot design using linear parameter-varying transformations. J. Guidance, Control, Dynamics, 16(2), 1993, pp. 256-261.

[33] Smith H.L. Monotone Dynamical Systems: An Introduction to the Theory of Competitive and Cooperative Systems, vol. 41 of Surveys and Monographs, AMS, Providence, 1995.

[34] Stamnes Ø.N., Aamo O.M., Kaasa G.-O. Redesign of adaptive observers for improved parameter identification in nonlinear systems. Automatica, 47(2), 2011, pp. 403-410.

[35] Tan W. Applications of Linear Parameter-Varying Control Theory. PhD thesis, Dept. of Mechanical Engineering, University of California at Berkeley, 1997.

[36] Theilliol D., Noura H., Ponsart J.-C. Fault diagnosis and accommodation of a three-tank system based on analytical redundancy. ISA Trans., 41, 2002, pp. 365-382.

[37] Xu A., Zhang Q. Residual Generation for Fault Diagnosis in Linear Time-Varying Systems. IEEE Trans. Autom. Control, 49(5), 2004, pp. 767-772.

[38] Yuan J. S.-C., Wonham W.M. Probing signals for model reference identification. IEEE Trans. Automat. Control, 22, 1977, pp. 530-538.

[39] Zemouche A., Boutayeb M. A unified adaptive observer synthesis method for a class of systems with both Lipschitz and monotone nonlinearities. Systems \&Control Let., 58(4), 2009, pp. 282-288.

[40] Zhang, Q. Adaptive observer for multiple-input-multiple-output (MIMO) linear time varying systems. IEEE Trans. Autom. Control, 47(3), 2002, pp. 525-529.

[41] Zolghadri A., Henry D., Monsion M. Design of nonlinear observers for fault diagnosis: a case study. Control Eng. Practice, 4(11), 1996, pp. 1535-1544. 